TUW-99-14

\title{
Hawking radiation from dilaton gravity in $1+1$ dimensions: a pedagogical review
}

\author{
W. Kummer円* \\ D. V. Vassilevich $\stackrel{\text { and }}{\dagger+}$ \\ ${ }^{1}$ Institut für Theoretische Physik, Technische Universität Wien \\ Wiedner Hauptstr. 8-10, A-1040 Wien \\ Austria \\ ${ }^{2}$ Institut für Theoretische Physik, Universität Leipzig, \\ Augustusplatz 10, D-04109 Leipzig, \\ Germany
}

\begin{abstract}
Hawking radiation in $d=4$ is regarded as a well understood quantum theoretical feature of Black Holes or of other geometric backgrounds with an event horizon. On the other hand, the dilaton theory emerging after spherical reduction and generalized dilaton theories only during the last years became the subject of numerous studies which unveiled a surprisingly difficult situation. Recently we have found some solution to the problem of Hawking flux in spherically reduced gravity which has the merit of using a minimal input. It leads to exact cancellation of negative contributions to this radiative flux, encountered in other approaches at infinity, so that our result asymptotically coincides with the one of minimally coupled scalars. The use of an integrated action is avoided although we have been able to present also that quantity in a closed expression. This short review also summarizes and critically discusses recent activities in this field, including the problem of "conformal frames" for the background and questions which seem to be open in our own approach as well as in others.
\end{abstract}

\section{Introduction}

The last years have seen an increased interest in dilaton theories, treated in $1+1$ dimensions. The main motivation to study such theories derives from the fact that spherical reduction of $D$-dimensional Einstein gravity (SRG) precisely generates a theory of this type [1].

\footnotetext{
${ }^{1 \star}$ email:wkummer@tph.tuwien.ac.at

$2 \dagger$ e-mail: Dmitri.Vassilevich@itp.uni-leipzig.de

$\ddagger$ On leave from Department of Theoretical Physics, St. Petersburg University, 198904 St. Petersburg, Russia
} 
Consider a line element

$$
(d s)^{2}=g_{\mu \nu} d x^{\mu} d x^{\nu}-\frac{4}{\lambda^{2}} e^{-\frac{4}{D-2} \phi}(d \Omega)^{2},
$$

where $d \Omega$ is the standard surface element on the $D-2$-dimensional unit sphere $S^{D-2}$. The dilaton field $\phi$ depends on the two first coordinates $x^{\mu}$ only. Then the $D$-dimensional Einstein-Hilbert action, after $\int d^{2} \Omega$ has been dropped, becomes the one of SRG

$$
\mathcal{L}_{S R G}=e^{-2 \phi} \sqrt{-g}\left(R+\frac{4(D-3)}{D-2}(\nabla \phi)^{2}-\frac{\lambda^{2}}{4}(D-2)(D-3) e^{\frac{4}{d-2} \phi}\right),
$$

which represents a particular dilaton theory with the Schwarzschild Black hole (SBH) in $D$ dimensions as its general classical solution.

As of 1991 also the special case $D \rightarrow \infty, \lambda^{2} D^{2} \rightarrow$ const. (dilaton Black Hole, $\mathrm{DBH})$ received particular attention [2]. Inspired by string theory [3] this model also exhibits a BH solution, although its singularity is null-complete [1. On the other hand, (2) allows a (classical) solution even when coupling to matter is introduced.

However, until quite recently in the latter case a quantum treatment of an action like (2) which went beyond the semiclassical calculations of the original literature was not known. In several recent papers 5 , the authors have shown (together with $\mathrm{H}$. Liebl) that a quantum treatment including full back-reaction for $2 D$ gravity can be formulated in which "geometry" is integrated out (trivially) and matter can be considered in a loop-wise expansion. Intriguing effects of "BH production" can be observed [6].

A necessary prerequisite for the plausibility of $2 D$ quantum gravity arguments of such a type is that a simpler consequence of Einstein theory in $D=4$ is consistently reproduced at the level of a SRG action like (2) or its generalizations. This simpler consequence is Hawking radiation from a fixed SBH background which is determined by the solution of (2). For a large $\mathrm{BH}$ $\left(m_{B H}>>m_{\text {Planck }}\right)$ this should be an excellent approximation. Of course, it neglects quantum effects from gravity.

Somewhat surprisingly until the seminal work of Mukhanov, Wipf and Zelnikov [7] no calculation to that effect had been performed - probably because no differences had been expected with respect to the well-known $D=4$ approach [8, 9. 10]. It was noted by the authors of [7] that technical difficulties arise, when the matter Lagrangian with minimal coupling of massless scalars $f$ in $D=4$ $(a=0,1,2,3)$

$$
{ }^{(4)} \mathcal{L}=\frac{1}{2}{\sqrt{-{ }^{(4)} g}}^{(4)} g^{a b}\left(\partial_{a} f\right)\left(\partial_{b} f\right)
$$

is spherically reduced. In its $2 D$ form a dependence on the dilaton field $\phi$ appears

$$
\mathcal{L}^{(n m)}=\frac{1}{2} e^{-2 \phi} \sqrt{-g} g^{\mu \nu}\left(\partial_{\mu} f\right)\left(\partial_{\nu} f\right)
$$


which must be taken into account carefully. Otherwise inevitably a negative flux of energy at infinity is related to the radiating $\mathrm{BH}$.

The problem resurfaced again in 1997 as a consequence of a paper by R. Bousso and S.W. Hawking [11 which contradicted [7] and work of the present authors together with H. Liebl [12], as well as others [13]. In 12] the problem had been considered for a generalization of (2) to the action [4]

$$
L=\int d^{2} x \sqrt{-g} e^{-2 \phi}\left(R+4 a(\nabla \phi)^{2}+B e^{2(1-a-b) \phi}\right) .
$$

of which (2) is the particular case $a=\frac{D-3}{D-2}, b=a-1$ and $B=-\frac{\lambda^{2}}{4}(D-2)(D-3)$.

This action not only covers SRG, but also the DBH [2]. For $a=1, b=0$, the Jackiw-Teitelboim model [14] $a=0, b=1$ is included. Mignemi [15] considers $a=1$ and all values of $b$. The models of ref. [16] correspond to $b=0, a \leq 1$. Lemos and Sa [17] discussed global solutions for $b=1-a$ and all values of $a$.

In [1] the global properties of all models of this type were analyzed in a systematic way (cf. the table Fig. 6 in the second ref. of [4]). The action (5) includes all $2 D$ theories with one singularity and one horizon. Only along the line $b=1-a$ in the interval $0<a<1$ (or $3<D<\infty$ ) the conditions of a "Minkowski ground state" theory [ $\mid 1$ are fulfilled, i.e. the presence of the singularity is tied to a non-vanishing Arnowitt-Deser-Misner (ADM) mass |18] which in $D=2$ is proportional to an absolutely (in space and time) conserved quantity, even when general matter interactions are included [19]. Also only for those models the conformal (Carter - Penrose) diagram coincides with the one of SRG.

Already before the study of Hawking radiation for nonminimally coupled scalars as in (4), where $\phi$ was even generalized to a function $\varphi(\phi)$, minimally coupled scalars had been considered on such backgrounds in this context [20]. Here - even for asymptotic de Sitter and Rindler space-times - no problems with the flux had arisen. But, as pointed out in 12 for nonminimal coupling a "straightforward" application of arguments, valid in $d=4$, to $D=2$ resulted in a negative flux at infinity - even when some mistaken conceptions regarding the conformal anomaly in 111 were corrected 21.

In $D=4$ two standard lines of arguments are used to derive the flux of Hawking radiation to infinity and its relation to Hawking-temperature, the temperature at the horizon seen by an asymptotic observer (we restrict ourselves to asymptotically flat situations, for simplicity).

The first - and most well-known - one has as its origin in a specific application of the Unruh effect [22, 9]: For two systems in relative accelerated motion the quantum vacuum of one system appears as a state with thermally distributed particles of a temperature which is proportional to the acceleration. Surface gravity, a geometric quantity determined by the normal derivative of the Killing norm at the (nondegenerate) horizon, in this way turns out to be proportional to the Hawking temperature 23]. On the other hand, the flux of radiation to infinity is computed from Bogoliubov coefficients which - during the the formation of a $\mathrm{BH}$ - relate incoming spherical waves before the collapse with 
outgoing ones from the neighbourhood of the horizon 8, 9]. This flux shows BH radiation with the $\mathrm{BH}$ behaving like a black body at the Hawking temperature.

Very soon it was realized that the formation of the $\mathrm{BH}$ is of relatively little importance for the bulk effect. What is really relevant are the null directions from the horizon to light-like infinity $\left(\mathcal{I}^{+}\right)$. Therefore, the second main approach due to Christensen and Fulling $(\mathrm{CF}) 10$ restricts itself mostly to that region. Integrating the conservation law of the energy momentum flux to infinity for minimally coupled scalars in $D=2$ the correct Hawking flux was obtained. Here the "quantum input" is the one loop quantum correction to the trace of the energy momentum (EM) tensor derived from (3) 3 and the assumption that the radiation flux should stay finite at the horizon (Unruh vacuum [9]), when considered in terms of global (Kruskal - Szekeres) coordinates. However, in the computation in $D=4$ several functions remain undetermined, even when all quantities are assumed to depend on time and one radial coordinate only.

In a calculation at the spherically reduced level $(D=2$, eq. (5)) clearly both these approaches should be viable - at least in principle. In addition, choosing the conformal gauge $g_{\mu \nu}=\eta_{\mu \nu} \exp (2 \rho)$ the dependence on a single function $\rho$ allows the functional integration of the trace anomaly to an effective action. The dependence of $\rho$ can be "covariantized" to the (nonlocal) Polyakov action 24] for scalars coupled minimally in $D=2$ (i.e. without the factor $\exp (-2 \phi)$ in (雨) or for some generalization thereof, if an additional dependence on the dilaton field exists. From that effective action the EM tensor and, as a consequence, also the flux to (asymptotically flat) infinity should be calculable. This line had been followed in ref. [7], where the authors realized that another non-Weyl-invariant piece must be added to the action which would be missed in a naive functional integration. However that piece could not be obtained exactly. Subsequently this problem has been focused further in [25] and 26] where the authors tried to construct the missing piece on the basis of general arguments.

All approaches which rely on an integrated effective action share a basic problem: The trace-anomaly is a local (UV) quantum effect. In order to exist as a mathematical object, the integrated effective action assumes vanishing fields at infinity. But just there the flux is calculated from it. Therefore, already for the case of minimally coupled scalars in general dilaton theories [20] the CF approach was used, as well as in the first application to nonminimally coupled scalars by the present authors 21]. In that paper the same EM conservation as in $D=4$ was assumed leading to an unacceptable negative flux for SRG.

Our recent attempt to achieve an exact treatment of this problem 27] in terms of a properly adapted $\mathrm{CF}$ approach has been the first successful one in the sense that it yielded the correct relation between Hawking temperature and flux at infinity. Exact cancellation of the negative flux against a piece derived from a "dilaton field anomaly" of the effective action was observed, leaving the asymptotic flux for minimal coupling. As a by-product also the full integrated action was obtained - but not used for this derivation, because of the problem

\footnotetext{
${ }^{3}$ a traceless EM tensor is necessary at the classical level. Such a tensor does not follow from (3), but requires there an additional term $-R \sqrt{-g} f^{2}$. For the Ricci-flat background of a $\mathrm{BH}$ such a term vanishes.
} 
mentioned above.

In Section 2 we collect definitions and formulas for the exact solution for the background, as determined by the action (5). Here we also take the opportunity to clarify some quite common misunderstandings regarding different "conformal frames" for BH-s [28], although the situation has been clear to several authors for some time 29]. As a warm-up in Section 3 we consider minimally coupled scalars in $D=2$ and describe $\zeta$-function regularisation to extract the trace (or "conformal") anomaly of the EM tensor. For nonminimal coupling (Section 4) the conservation of the EM-tensor in the presence of a dilaton field receives a further contribution [25], [26], which requires another input, the variation of the effective action with respect to the dilaton field. The trace anomaly may be calculated for the generalized nonminimal coupling $\phi \rightarrow \varphi(\phi)$ in (14) and for a general dilaton dependent quantum measure 112]. The additional piece, the " $\phi$ anomaly", no longer follows from multiplicative variation of the differential operator in the scalar action. Therefore a new method had to be developed to extract that quantity [27]. The computation of the Hawking flux to infinity and the discussion of renormalization which is important for the flux at finite distances in the $\mathrm{CF}$ approach conclude this section.

By formal (functional) integration also the full effective action is obtained (Section 5). In its covariantized version it represents the generalization of the Polyakov action for generic dilaton theories [27].

Section 6 is devoted to a discussion of the place where effects from backscattering may enter the $2 \mathrm{D}$ approach.

We summarize our results in Section 7 where also related other work and our own results are critically reviewed. Open problems and directions for further research are mentioned.

\section{Geometric background with dilaton fields}

All classical solutions of the models ([5) are known for some time [30]. They can be obtained in an especially easy way in the first order gravity version of such models and using Cartan variables in the light-cone gauge which corresponds to the Eddington-Finkelstein gauge for the metric [19], [4]. Letting $\phi$ represent one of the coordinates, the line element reads 4

$$
(d s)^{2}=g(\phi)\left(2 d v d \phi+l(\phi) d v^{2}\right),
$$

with

$$
\begin{gathered}
g(\phi)=e^{-2(1-a) \phi} \\
b \neq-1: \quad l(\phi)=\frac{e^{2 \phi}}{8}\left(C-\frac{2 B}{b+1} e^{-2(b+1) \phi}\right), \\
b=-1: \quad l(\phi)=\frac{e^{2 \phi}}{8}(\tilde{C}+4 B \phi), \tilde{C}=C-2 B \ln 2 .
\end{gathered}
$$

$C$ is an integration constant, related to the ADM-mass for the subset of such models, which are asymptotically flat. 
Not all the models (5) are suitable for analyzing Hawking radiation. By definition, the latter one is the amount of energy radiated by a black hole to the asymptotic region. That region must represent an "empty space" in the solution. There are three subclasses of the models (6) for which such "empty space" solutions are well defined. These are the asymptotically Minkowski $(b=a-1)$, asymptotically Rindler $(b=0)$, and asymptotically de Sitter $(b=1-a)$ models.

For minimally coupled scalars on such a background, all three cases were discussed in [20]. Here we concentrate only on the first one which includes the D-dimensional BH-s. Bringing the solutions (7) - (9) to the generalized Schwarzschild form 20] we obtain

$$
(d s)^{2}=L(u) d t^{2}-\frac{d u^{2}}{L(u)} .
$$

Asymptotically Minkowski models are more physically transparent, so we use them as our main example. There we have

$$
L(u)=1-\left(\frac{u_{h}}{u}\right)^{\frac{a}{1-a}}, \quad \phi(u)=-\frac{1}{2(1-a)} \ln (2(1-a) u) .
$$

$u_{h}$ is the coordinate of the horizon, defined through the equation $l\left(\phi\left(u_{h}\right)\right)=0$. For $a=\frac{D-3}{D-2}$ one obtains the familiar Schwarzschild BH in $D$ dimensions $\left(u_{h}=\right.$ $2 M_{A D M}$ in $\left.D=4\right)$.

We shall also use the conformal version of the metric $\left(x^{ \pm}=\tau \pm z\right)$

$$
(d s)^{2}=L(u) d x^{+} d x^{-}=e^{2 \rho(u)} d x^{+} d x^{-}, \quad d u=L(u) d z .
$$

It is essential, that variables are rescaled appropriately so that the function $L(u)$ as in (11) tends to unity at the asymptotic region. In this manner the introduction of a rescaling factor for the Hawking temperature $T_{H}$ is avoided. As $L(u)$ is nothing but the Killing norm, $T_{H}$ is related to the surface gravity 23] 31]

$$
T_{H}=\frac{1}{4 \pi} L^{\prime}(u)=\frac{1}{4 \pi u_{h}} \frac{a}{1-a}=\frac{D-3}{4 \pi u_{h}},
$$

where the last expression refers to the $D$-dimensional SBH.

In this short review we deal only with models describing a single Schwarzschildlike BH. More complex solutions as, for example, exotic multiple horizon Nariai BH-s [32] will not be considered, since they are regarded as a "pathology" even by the authors of corresponding research papers (see the last paper of ref. [32]). They also clearly are not covered by our $2 d$ approach.

Over the last years comparisons of Hawking radiation (and of quantized versions of $2 d$ dilaton theories) in models differing by conformal transformations

$$
\begin{aligned}
& g_{\mu \nu}=e^{-2 \xi} \widetilde{g}_{\mu \nu} \\
& \sqrt{-g} R=\sqrt{-\widetilde{g}} \widetilde{R}+2 \partial_{\mu}\left[\sqrt{-\widetilde{g}} \widetilde{g}^{\mu \nu} \partial_{\nu} \xi\right]
\end{aligned}
$$


can be found in the literature [28. Indeed, it is tempting to choose $\xi(\phi)$ in (14) in such a way that the kinetic term $(\nabla \phi)^{2}$ in (2) or in the most general version of a dilaton theory $(X=-2 \exp (-2 \phi))$

$$
\mathcal{L}_{d i l}=\sqrt{-g}\left[-\frac{X R}{2}+U(X)(\nabla X)^{2}-V(X)\right]
$$

is made to disappear. As a strategy to obtain a solution to (16) in an easier manner this is certainly legitimate - just as the introduction of a corresponding canonical transformation in the Hamiltonian version of such a theory. But it should be kept in mind that (16), the geometric part of the action, is not invariant under (14) (as it would be for Weyl-invariance in the trivial string case). It is crucial, therefore, that the same transformation of fields is performed in the "less visible" parts of the theory. The global properties of manifolds - like (11) for the $\mathrm{BH}$ - are determined by the continuation of coordinate patches through incomplete boundaries where no singularities are located. "Incompleteness" means that at least one null or non-null geodesic reaches that boundary at a finite value of the affine parameter. For the BH (and for manifolds with more complicated topology as treated by Klösch and Strobl [19]) this procedure ultimately leads to - sometimes quite complicated - (global) Penrose diagrams. Clearly the geodesics for the manifold involving a certain $g_{\mu \nu}$ are to be computed for the same metric. Equivalently, those geodesics can be considered to originate from an additional piece of the action describing the motion of a (point) test mass in the background geometry. But these geodesics describing the global properties of the manifolds must not be confused with the couplings to matter fields as in (4) which, of course, remain unchanged by the formal transition from $g$ to $\widetilde{g}$.

Now consider the same model after the transformation (14), (15). It is obvious from the argument above that also in the geodesic equation $g$ must be transformed to $\widetilde{g}$ ! The derivation of $T_{H}$ from the surface gravity, leading to (12) had been based upon $g$. Doing all the calculations in terms of $\widetilde{g}$, therefore, should be consistent - as long as no obstructions are implied by the transformation (14). Because when the map $g \rightarrow \widetilde{g}$ is not isomorphic, or if it even introduces a singularity, the $\widetilde{g}$ system will become correspondingly complicated - if treated correctly! Precisely this happens e.g. in the case of the DBH [3] when the kinetic $(\nabla \phi)^{2}$ - term of the dilaton field is transformed away. For that model the $\widetilde{g}$ - theory exhibits a geometric part with Rindler geometry (constant acceleration) and the BH-like singularity disappears altogether - which is clearly a completely different theory.

We stress that the experience from ordinary field theory in a fixed (Minkowski) background is misleading. There a redefinition of fields does not change the (Minkowski) manifold, upon which these fields live. For gravity the situation is fundamentally different. Here the "field" (geometric variable: $g_{\mu \nu}$, dilaton field...) at the same time defines the manifold. Therefore even locally (cf. the change of the curvature, eq. (15)!) the transformed theory refers to a different manifold.

As a special illustration consider the action (16) with $U=0$. Its exact solutions for the line element is [19] (6) with $g(\phi)=1$ and, in terms of the 
variable $X=u$ already introduced in (10), (11),

$$
l(u)=\mathcal{C}-\int_{u o}^{u} d y V(y) d y=\mathcal{C}-w(u),
$$

where $C$ is the integration constant labelling a certain solution. It is that quantity which changes under the influence of interacting matter 19. Such a model can only describe "eternal" singularities, because they must appear in $w(u)$ and are determined by the (given) parameters of the action. Some instantaneous influx of matter only shifts the position of the horizon and has no effect upon the singularity (-ies). Outside the horizon we may identify $u$ with the "radius". The surface gravity allows an interpretation as a Hawking temperature at a horizon, if $w(\infty) \rightarrow 0$, i.e. if the metric (6) becomes asymptotically flat f $^{\text {. Af- }}$ ter a conformal transformation (14) with $w(u)=\exp 2 \xi$ and after introducing a new coordinate $d \tilde{u}=d u w^{-1}(u)$ the line element $(d s)^{2}$ with (17) and the new one

$$
\begin{aligned}
& (d \widetilde{s})^{2}=2 d \widetilde{u} d v+\widetilde{l}(\widetilde{u})(d v)^{2} \\
& \widetilde{l}(\widetilde{u})=\frac{\mathcal{C}}{w(u(\widetilde{u}))}-1
\end{aligned}
$$

can be compared. Clearly $(18)$ with $(\sqrt{19})$ will be solutions of a model $(16)$ with $U \neq 0$. But in terms of the new radial variable $\widetilde{u}$ now obviously a new singularity develops at $\widetilde{u} \rightarrow \infty$ (or $u \rightarrow-\infty$, depending on the sign of $w$ ). On the other hand, the original "eternal" singularity in $w(u)$ generically will disappear, but new ones will develop from the zeros of the latter quantity. The original horizon at $u=u_{h}$ in (17) still will be a horizon (zero) of (19), but it will be related to quite different singularities, and - if such a region exists at all for the new model (18)! - a different asymptotically flat regime at the, in general, opposite side of the horizon. A theory with generic metric (19) clearly has a flat ground-state $(\mathcal{C}=0)$. SBH and DBH are special cases. In fact, the Lagrangian (16) for a general dilaton theory with this "Minkowski ground state" property, where $U$ depends in a specific way on $V$, can be written down easily .

Additional complications by the field transformation (14), (15) arise in the quantum version of such theories . E..g. due to the change of global properties the definition of "asymptotic states" for some quantum gravity S-matrix ( a horrendously difficult problem by itself) becomes completely undefined. Thus "quantization" in terms of $g$ resp. $\widetilde{g}$ (or using even locally well-defined canonical transformations in a Hamiltonian formulation) refers to completely different quantum field theories [29].

In view of these comments it cannot come as a surprise that the ADM mass of a black hole is not conformally invariant [33, 20]. As in the case of $4 \mathrm{D}$ Einstein gravity, in order to define the ADM mass unambiguously it is crucial to choose a proper asymptotic behavior of the metric [34]. Again, it is important which metric, $g$ or $\tilde{g}$, is the "physical" one. The very existence of the ADM

\footnotetext{
${ }^{4}$ asymptotics may be discussed in the similar manner.
} 
mass means that the action is not invariant under the diffeomorphisms which do not vanish at the boundary. Another example of conformal non-invariance of physical processes in $2 D$ dilaton gravity has been given recently in [35].

\section{$3 \quad$ Hawking radiation from minimally coupled scalars}

\subsection{EM tensor}

The EM tensor for minimally coupled scalars is defined from the action $W^{(\min )}$ with the Langrangian (3), dropping the dilaton factor $e^{-2 \phi}$ :

$$
T_{\mu \nu}^{(\min )}=\frac{2}{\sqrt{-g}} \frac{\delta W^{(m i n)}}{\delta g^{\mu \nu}}=\left(\partial_{\mu} f\right)\left(\partial_{\nu} f\right)-\frac{g_{\mu \nu}}{2}\left(\partial^{\alpha} f\right)\left(\partial_{\alpha} f\right)
$$

From diffeomorphism invariance of the matter action (see below for the more general argument, if dilaton fields are present in the Langrangian (4D), taking the matter fields on-shell, the usual EM conservation follows (we drop the superscript (min) for simplicity in this section):

$$
\nabla_{\mu} T^{\mu \nu}=0 .
$$

In the conformal gauge (12) only the components $\Gamma_{++}{ }^{+}=2 \partial_{+} \rho, \Gamma_{--}{ }^{-}=$ $2 \partial_{-} \rho$ are nonzero. Thus equation (21) for $\nu=+$ reads

$$
\partial_{+} T_{--}+\partial_{-} T_{+-}-2\left(\partial_{-} \rho\right) T_{+-}=0 .
$$

According to (11), (12) the background depends on the variable $u$ alone. Thus derivatives of light cone coordinates, acting on functions of $u$ become

$$
\partial_{+}=-\partial_{-}=\frac{1}{2} \partial_{z}=-\frac{1}{2} L(u) \partial_{u},
$$

and (22) turns into a simple first order differential equation in $z$

$$
\partial_{z} T_{--}=\left[\partial_{z}-2\left(\partial_{z} \rho\right)\right] T_{+-},
$$

which may be integrated easily for the flux component $T_{--}$if $T_{+-}$is known. We will be interested in the flux at infinity where the space should become flat $\left(g_{\mu \nu} \rightarrow \eta_{\mu \nu}\right)$. In light-cone coordinates $x^{ \pm}=x^{\circ} \pm x^{1}=\tau \pm z$ the flux orthogonal to the lines $x^{-}=$const. in the direction of outgoing waves is just $T_{--}$. On the other hand, $T_{+-}$is nothing else but the trace of $T_{\mu \nu}$,

$$
T_{\mu}^{\mu}=g^{\mu \nu} T_{\mu \nu}=4 e^{-2 \rho} T_{+-},
$$

which vanishes identically for the classical action according to (20). A solution for $f$ in conformal gauge still obeys the free wave equation $\partial_{+} \partial_{-} f=0$ with solutions $f=F_{+}\left(x^{+}\right)+F_{-}\left(x^{-}\right)$. Thus the EM term (20) at the classical level at large distances $f=F_{ \pm}$is of the form

$$
\left.T_{\mu \nu}^{ \pm}\right|_{a s}=a_{ \pm}\left(\begin{array}{ll}
1 & \pm 1 \\
\pm 1 & 1
\end{array}\right)
$$


where $a_{ \pm} \propto\left(F_{ \pm}^{\prime}\right)^{2}$. Classically no flux to infinity should occur from a BH, therefore $a_{ \pm}=0$ which is consistent with the trivial solution $f=0$.

On the other hand, if Hawking radiation exists, it must be a quantum effect. Then the constant $a_{ \pm}=\frac{\pi}{12} T_{H}^{2}$ (the Stefan-Boltzmann law in $D=2$ ) in front of (26) can be determined by comparison with the EM tensor $T_{\mu \nu}^{(e)}$ for black body radiation in $D=2$ in equilibrium with a heat bath, where the pressure equals the radiation density,

$$
\left.T_{\mu \nu}^{(e)}\right|_{a s}=\frac{\pi}{6} T_{H}^{2}\left(\begin{array}{ll}
1 & 0 \\
0 & 1
\end{array}\right) .
$$

The equilibrium EM tensor from (26) with $a_{+}=a_{-}$must be $T^{+}+T^{-}$ [10]. In any case, for the determination of the flux at infinity it is enough to know one component, like $T_{--}$introduced above. As emphasized already, minimally coupled scalars in $D=2$ conformal gauge (12) also at nonasymptotic distances are still determined by a free wave equation. They do not "feel" the geometry. Therefore $\left.T_{\mu \nu}\right|_{\text {as }}$ will coincide with the asymptotic value of $T_{\mu \nu}$ as calculated from the classical action. This is not the case in $D=4$ where a complication from emission - absorption coefficients ("grey-factors") arises [10], [7, and, as will be sketched below (Section 6), also introduces complications in the spherically reduced case, i.e. for nonminimally (dilaton coupled) scalars.

If first order quantum loops of the scalar field are taken into account, the full (effective) action $W$ will acquire a contribution with nonvanishing trace (25). As it breaks the conformal symmetry of the scalar interaction - note, however that the geometric part of the action is not conformally invariant! -, this new term will be called the "conformal anomaly".

We now interpret (24) to represent the one-loop quantum contributions on both sides of this equation. If the conformal anomaly is known, it may be integrated outside the horizon $u_{h} \leq u(-\infty \leq z)$ all the way to its asymptotic value $\left.T_{--}\right|_{u \rightarrow \infty}=\left.T_{--}\right|_{a s}$. Beside $T_{+-}$the only input then is an integration constant which determines $\left.T_{--}\right|_{u_{h}}=t_{--}$.

Before discussing different choices of the boundary values for $T_{--} \mid{ }_{u_{h}}$ which are related to different "quantum vacua" 36, [37, [9] it is important to understand the behaviour of $T_{--} \mid{ }_{u_{h}}$ in terms of global (Kruskal - Szekeres) coordinates. For a nondegenerate Killing horizon in the neighbourhood of $u \approx u_{h}$ the Killing norm has a simple zero. Hence in (5)

$$
(d s)^{2} \approx 2 d v\left(d u+a\left(u-u_{h}\right) d v\right)
$$

with some constant $a$, proportional to the surface gravity (which in the following will be set equal to 1$)$, in the Killing norm $l(u) \approx a\left(u-u_{h}\right)$. The variable $u$ is taken to be the one which is related to the conformal radial variable $z$ (tortoise coordinate) in (11),

$$
z=\int^{u} \frac{d y}{l(y)} \approx \ln \left(u-u_{h}\right) \approx \ln l(u)
$$


so that in conformal gauge (12) $\left(x^{-}=\tau-z\right)$

$$
(d s)^{2} \approx e^{z} d x^{+} d x^{-}=e^{\left(x^{+}-x^{-}\right)} d x^{+} d x^{-} .
$$

Global conformal coordinates $\left(d s^{2} \approx d \bar{x}^{+} d \bar{x}^{-}\right)$near the horizon are introduced by

$$
\bar{x}^{ \pm}= \pm 2 e^{ \pm x^{ \pm} / 2},
$$

so that the components of $\bar{T}_{\mu \nu}$ by reparametrization $x^{ \pm} \rightarrow \bar{x}^{ \pm}$become

$$
\begin{aligned}
& \bar{T}_{--}=\frac{4}{\left(y^{-}\right)^{2}} T_{--}, \\
& \bar{T}_{+-}=-\frac{4}{y^{+} y^{-}} T_{+-}, \\
& \bar{T}_{++}=\frac{4}{\left(y^{+}\right)^{2}} T_{++} .
\end{aligned}
$$

For the factor of $T_{--}$in $\bar{T}_{--}$at fixed $x^{+}=\tau+z$ we obtain

$$
\left(y^{-}\right)^{-2}=\frac{1}{4} e^{(\tau-z)}=\frac{1}{4} e^{\left(x^{+}-2 z\right)} \propto\left(u-u_{h}\right)^{-2} .
$$

In a similar way the factors in $\bar{T}_{-+}$and $\bar{T}_{++}$are found to be $\left(u-u_{h}\right)^{-1}$ and a constant, respectively.

Before it had been realized that BH-s may radiate, it had seemed natural enough to assume that $\left.T_{--}\right|_{a s} \rightarrow 0$ ( Boulware vacuum [36|). This leads to a divergent $\bar{T}_{--}$at the horizon [10]. Another proposal considering the BH-system in thermal equilibrium with a heat bath [37, 23 assumed regularity of $T_{\mu \nu}$ at the past and at the future event horizon. In this case (27), the sum of ingoing and outgoing fluxes at infinity would be relevant. This is not the situation we are interested in. However, from the argument above which is restricted to the region between the future horizon and positive null infinity $\mathcal{I}^{+}(u=\infty)$ the choice $\left.T_{--}\right|_{u \approx u_{h}}=O\left(u-u_{h}\right)^{2}$, providing a finite flux in global coordinates at the horizon [9], seems the most obvious one.

Indeed in ref. [10] it was shown that the Unruh vacuum for $D=2$ minimally coupled scalars is consistent. It precisely relates the Hawking temperature $T_{H}=\left(4 \pi u_{h}\right)^{-1}$ to the asymptotic flux (21) when the (known) conformal anomaly $T_{+-}$(see below) is used.

\subsection{Conformal anomaly}

The contribution of the scalar loop to the trace of the EM tensor in an external gravitation field is most effectively calculated in the heat-kernel approach [38], 339]. 
The classical action from (5) for minimal coupling by partial integration can be written as

$$
S^{(m i n)}=\frac{1}{2} \int \sqrt[4]{-g} f A^{(\min )} \sqrt[4]{-g} f
$$

with the differential operator

$$
A^{(\min )}=-g^{\mu \nu} \nabla_{\mu} \nabla_{\nu}
$$

pulling determinants of the metric through the covariant derivative $\nabla_{\mu}$. In the path integral for the one-loop effective action $W$ we turn to the Euclidean region $\sqrt{-g} \rightarrow \sqrt{g} 40$

$$
\exp W=\int(d f \sqrt[4]{g}) \exp S,
$$

where the factor $\sqrt[4]{g}$ in the measure guarantees covariance of the Gaussian measure

$$
\begin{aligned}
& \int(\sqrt[4]{g} d f) \exp \langle f, f\rangle=\text { invariant } \\
& \left\langle f_{1}, f_{2}\right\rangle=\int d^{2} x \sqrt{g} f_{1} f_{2}
\end{aligned}
$$

The result of the integral (36) is the effective action, given by the functional determinant of $A^{(\mathrm{min})}=A$ 40

$$
W=\ln (\operatorname{det} A)^{1 / 2}=\frac{1}{2} \operatorname{Tr} \ln A .
$$

In the zeta-function regularization 41 a power of $A$ instead of the logarithm is considered:

$$
W=-\frac{1}{2} \zeta_{A}^{\prime}(0), \quad \zeta_{A}(s)=\operatorname{Tr}\left(A^{-s}\right)
$$

Prime denotes differentiation with respect to $s$. No analytic general results exist for the finite part of (40), but they do for certain variations of this quantity. We start our analysis with the calculation for an arbitrary conformally covariant operator $A$.

Conformal covariance means that under an infinitesimal conformal transformation of the metric $\delta g_{\mu \nu}=\delta k(x) g_{\mu \nu} \delta g^{\mu \nu}=-\delta k g^{\mu \nu}$ the operator $A$ transforms as

$$
\delta A=-\delta k A
$$

The same conformal transformation in the effective action produces the quantum contribution to trace of the energy-momentum tensor (20)

$$
\delta W=\frac{1}{2} \int d^{2} x \sqrt{g} \delta g^{\mu \nu} T_{\mu \nu}=-\frac{1}{2} \int d^{2} x \sqrt{g} \delta k(x) T_{\mu}^{\mu}(x) .
$$


The variation of the zeta function with respect to a certain parameter or field in $A$ is related to the one of the operator $A$ [42] [39]:

$$
\delta \zeta_{A}(s)=-s \operatorname{Tr}\left((\delta A) A^{-1-s}\right)
$$

Due to conformal covariance (41) the powers of $A$ in (43) recombine back into $A^{-s}$. Thus with the definition of a generalized $\zeta$-function [38] [39]

$$
\zeta(s \mid \delta k, A)=\operatorname{Tr}\left(\delta k A^{-s}\right)
$$

the variation in (42) can be identified with

$$
\delta W=-\frac{1}{2} \zeta(0 \mid \delta k, A) .
$$

We thus encounter a multiplicative variation of the operator $A$. For such variations of $\zeta_{A}$ the general result is known.

Combining (45) and (42) yields

$$
\zeta(0 \mid \delta k, A)=\int d^{2} x \sqrt{g} \delta k(x) T_{\mu}^{\mu}(x) .
$$

By a Mellin transformation one can show that $\zeta(0 \mid \delta k, A)=a_{1}(\delta k, A)$ [39], where $a_{1}$ is defined as a coefficient in a small $t$ asymptotic expansion of the heat kernel containing $F$ multiplicatively:

$$
\operatorname{Tr}(F \exp (-A t))=\sum_{n} a_{n}(F, A) t^{n-1}
$$

To evaluate the form of $a_{1}$ we use the standard method [39]. We assume that $A$ as in our application is an operator of Laplace type. This means that one can represent it as

$$
A=-\left(\hat{g}^{\mu \nu} \hat{D}_{\mu} \hat{D}_{\nu}+E\right)
$$

with a suitable choice of the metric $\hat{g}^{\mu \nu}$, a related covariant derivative $\hat{D}_{\mu}$, and an endomorphism $E$. Then the result for $a_{1}$ can be simply taken from the literature [39], returning to Minkowski space $(\sqrt{g} \rightarrow \sqrt{-g})$

$$
a_{1}(\delta k, A)=\frac{1}{24 \pi} \operatorname{tr} \int d^{2} x \sqrt{-\hat{g}} \delta k(\hat{R}+6 E) .
$$

Here tr denotes ordinary trace over all matrix indices (if there are any in $A$ ). $\hat{R}$ is the scalar curvature for the metric $\hat{g}$.

For $A=A^{(\min )}$ in (40), $g^{\mu \nu}=\hat{g}^{\mu \nu}, E=0$ the conformal anomaly follows immediately from (49) and (42) with the definitions (20) for the EM tensor now used for the effective action (39) 


$$
T_{\mu}^{\mu}=\frac{1}{\sqrt{g}} \frac{\delta}{\delta k} a_{1}\left(\delta k, A^{(\min )}\right)=\frac{1}{24 \pi} R .
$$

The Ricci scalar $R$ is most easily computed for the conformal gauge by means of the identity (15) $\left(\xi=-\rho, \widetilde{g}_{\mu \nu}=\eta_{\mu \nu}, \widetilde{R}=0\right)$

$$
R=-\partial_{+} \partial_{-} \rho,
$$

yielding the $(+-)$ component of the energy-momentum tensor (cf. (25))

$$
T_{+-}^{(\min )}=-\frac{1}{12 \pi} \partial_{+} \partial_{-} \rho=\frac{1}{48 \pi} \partial_{z}^{2} \rho
$$

\subsection{Radiative flux}

Solving eq. (27) the radiative flux is obtained with the anomaly (52)

$$
T_{--}^{(\min )}=\frac{1}{48 \pi}\left[\partial_{z}^{2} \rho-\left(\partial_{z} \rho\right)^{2}\right]+t_{--},
$$

where $t_{--}$is the integration constant.

In terms of (12) this may be rewritten as

$$
T_{--}^{(m i n)}=\frac{1}{48 \pi}\left[\frac{L^{\prime \prime} L}{2}-\frac{L^{\prime 2}}{4}\right]+t_{--} .
$$

The behaviour of $L(u)$ near $u \approx u_{h}$ can be read off from the exact result for a $\mathrm{BH}$ background

$$
T_{--}^{[m i n]}=t_{--}-\frac{1}{192 \pi u_{h}^{2}}+\frac{\left(u-u_{h}\right)^{2}}{192 \pi u^{4} u_{h}^{2}}\left(u^{2}+2 u u_{h}+3 u_{h}^{2}\right) .
$$

One observes that a finite flux $\bar{T}_{--}$in global coordinates (32) at the horizon is indeed provided by fixing $t_{--}$alone to cancel the first term. On the other hand, the asymptotic flux at $u \rightarrow \infty$ is just given by the same expression as $t_{--}$when the other terms in (54) tend to zero,

$$
\left.T_{--}^{(m i n)}\right|_{\text {asymp }}=t_{--}=\frac{\pi}{12} T_{H}^{2},
$$

a result which is in perfect agreement with (26).

One can show 20 that the expression (56) for $T_{--}$in terms of the Hawking temperature is valid also for asymptotically Rindler and de Sitter models. In this connection we emphasize that the calculation of the Hawking flux is sensitive to the choice of the coordinate system. It is essential that $\rho \rightarrow 0$ in the asymptotic region. For $\rho \rightarrow$ const $\neq 0$ the flux is measured using time and length scales different from the ones used to measure the $\mathrm{BH}$ mass. If $\rho$ does not tend to a constant in the asymptotic region, the observer connected with such a coordinate 
system measures a mixture of Hawking and Unruh radiation. This happened in a recent calculation [43] for AdS BH-s, where the puzzling result of zero Hawking flux was obtained. Note, that due to the condition $\phi=0$ used in that reference to obtain solutions of the quantum corrected field equations the properly normalized (classical) ADM mass (cf. eq (14) in [20) would be zero.

So far in this section only models with one horizon were discussed. But the only necessary input for the method employed, the integration of the EM conservation in the sense of ref. 10] between the future event horizon and (flat) null-infinity $\mathcal{I}^{+}$applies equally well for all dilaton theories (16) provided their Killing norm $L(u)$ in the interval $u_{h} \leq u \leq \infty$ also behaves like (11), i.e. $L(\infty)=$ $L\left(u_{h}\right)=0$ when $u_{h}$ is the largest (simple) zero. That horizon may protect more than one singularity - or even none at all. We just note that within the first order gravity formulation of $2 D$ theories 19 it is straightforward to "design" such models easily by appropriate choices of $U(X)$ and $V(X)$ in (16), where $U(X)$ corresponds to a "torsion term" in the equivalent Cartan theory.

\section{Nonminimally coupled scalars}

As explained in the Introduction this case should be the closest one to the situation in (higher dimensional) Einstein gravity. Indeed the spherically reduced action (2) together with (4) exactly reproduces the same equations of motion, which are obtained from the variation of the Einstein-Hilbert action in $D$ dimensions when the dependence of the metric on $x^{\mu}=(t, r)$ alone, as in (11), is assumed [44]. This is not a trivial result: e.g. reducing with an ansatz of a "warped" metric does not allow the definition of a reduced Lagrangian from which the entire set of e.o.m.-s follow 45].

\subsection{EM tensor}

With the same definition (20) of the EM tensor $T_{\mu \nu}^{(n m)}$ for the nonminimal case, in the presence of an additional dilaton field we obtain at the classical level $T_{\mu \nu}^{(n m)}=e^{-2 \phi} T_{\mu \nu}^{(\min )}$. The factor $e^{-2 \phi}$ is nothing else but the (for $D \neq 4$ appropriately redefined) scaling factor or (radius) ${ }^{2}$ (cf. (1) for SRG). If interpreted with respect to the unreduced dimensional level, the flux is multiplied by the area of the sphere $S^{D-2}$ (remember that $\int d^{2} \Omega$ has been integrated out) - as it should be. In the present case where $W^{(n m)}=W^{(n m)}\left(g_{\mu \nu}, f, \phi\right)$ the conservation law (24) must be modified. By construction the matter field action is invariant under the diffeomorphism transformations

$$
\begin{aligned}
\delta g_{\mu \nu} & =\nabla_{\mu} \xi_{\nu}+\nabla_{\nu} \xi_{\mu} \\
\delta \phi & =\xi^{\nu} \partial_{\nu} \phi \\
\delta f & =\xi^{\nu} \partial_{\nu} f
\end{aligned}
$$

where $\phi$ denotes either the dilaton field or any local function thereof. By applying the transformations (57) to the nonminimal action $W^{(m n)}$ one obtains 
instead of (21)

$$
\nabla^{\mu} T_{\mu \nu}^{(n m)}=-\left(\partial_{\nu} \phi\right) \frac{1}{\sqrt{-g}} \frac{\delta W^{(n m)}}{\delta \phi}
$$

where a term containing $\delta W^{(m n)} / \delta f$ has been dropped on the r.h.s., when we assume the fields $f$ to be on-shell classically. Having integrated out the fluctuation of these fields in the one loop order also no contribution can come from those when (58) is applied to the effective action. When Hawking radiation is to be studied from (58) we shall assume that we start from a situation where no classical field $f$ is present, although some modifications will turn out to be necessary in a more detailed consideration (see Section 6). Then the r.h.s. of (58) vanishes for its classical contribution. As $T^{(n m)}$ also has a classically vanishing trace the situation is much like the minimal case (53), except for the fact that beside the conformal anomaly $T_{+-}^{(n m)}$ now also another term (" $\phi$-anomaly") appears together with contributions from the 1-loop version of (58), viz.

$$
\partial_{+} T_{--}^{(n m)}=-\left(\partial_{-}-2\left(\partial_{-} \rho\right)\right) T_{+-}^{(n m)}+\frac{\left(\partial_{-} \phi\right)}{\sqrt{-\eta}} \frac{\delta W^{(n m)}}{\delta \phi}
$$

or $(\mathrm{cf} .(23))$

$$
\partial_{z} T_{--}^{(n m)}=\left(\partial_{z}-2\left(\partial_{z} \rho\right)\right) T_{+-}^{(n m)}-\frac{\partial_{z} \phi}{\sqrt{-\eta}} \frac{\delta W^{(n m)}}{\delta \phi} .
$$

The last term in (60) had not been taken into account in [12].

Separating in $T_{+-}^{(n m)}$ the contribution of minimal coupling from the additional $T_{+-}^{(d i l)}$ which appears for non-vanishing dilaton fields

$$
T_{+-}^{(n m)}=T_{+-}^{(\min )}+T_{+-}^{(d i l)}
$$

in the total $T_{--}^{(\mathrm{nm})}$ from integration of 60 we obtain three pieces where the first one is known from (53):

$$
\begin{aligned}
& T_{--}^{(n m)}=T_{--}^{(\min )}+T_{--}^{(d i l)}+T_{--}^{(\phi)} \\
& T_{--}^{(d i l)}=\int_{-\infty}^{z}\left[\left(\partial_{z}-2\left(\partial_{z} \rho\right)\right) T_{+-}^{(d i l)}\right]_{z^{\prime}} d z^{\prime} \\
& T_{--}^{(\phi)}=\int_{-\infty}^{z} d z^{\prime}\left(\partial_{z^{\prime}} \phi\right)\left(\frac{1}{\sqrt{-\eta}} \frac{\delta W^{(n m)}}{\delta \phi}\right)_{z^{\prime}} .
\end{aligned}
$$

All integrals start at the horizon $\left(z=-\infty\right.$ or $\left.u=u_{h}\right)$. By analogy with the minimal case $\left.T_{--}\right|_{u_{h}}=0$ is assumed and, therefore, the overall integration constant $t_{--}$has been dropped. 


\subsection{Conformal anomaly}

We again follow the steps outlined in section 3.3, but starting from the nonminimal Lagrangian (田). This will change the differential operator $A$ to be used in (39).

A less trivial observation is that the dilaton field $\phi$ enters also the path integral measure (31). This happens because the scalar product for the $s$-wave modes in $D$ dimensions contains $\phi$, so that instead of (38)

$$
<f_{1}, f_{2}>=\int d^{2} x \sqrt{-g} e^{-2 \phi} f_{1} f_{2}
$$

holds. Therefore, only the redefined fields fields $\tilde{f}=e^{-\phi} f$ in two dimensions have a scalar product like (38). Consequently, $\tilde{f}$ possesses the Gaussian path integral measure (37). In the following we allow for a more general coupling and a more general path integral measure. To this end we replace $\phi$ by $\varphi(\phi)$ in the action (5) and $\phi$ by $\psi(\phi)$ in the measure, assuming that $\varphi$ and $\psi$ are some local functions of the dilaton.

Expressing the classical action corresponding to the Lagrangian (5) with $\phi \rightarrow \varphi(\phi)$ in terms of the field $\tilde{f}$ yields the action

$$
S^{(n m)}=\frac{1}{2} \int \sqrt{-g} d^{2} x \tilde{f} A^{(n m)} \tilde{f},
$$

containing the operator

$$
\begin{aligned}
A^{(n m)}= & -e^{-2 \varphi+2 \psi} g^{\mu \nu}\left(\nabla_{\mu} \nabla_{\nu}+2\left(\psi_{, \mu}-\varphi_{, \mu}\right) \nabla_{\nu}+\psi_{, \mu \nu}-\right. \\
& \left.-2 \varphi_{, \mu} \psi_{, \nu}+\psi_{, \mu} \psi_{, \nu}\right) .
\end{aligned}
$$

Thus the basic quantities entering the standard form (48) of the Laplace type operator are

$$
\hat{g}^{\mu \nu}=e^{-2 \varphi+2 \psi} g^{\mu \nu}, \quad E=\hat{g}^{\mu \nu}\left(-\varphi_{, \mu} \varphi_{, \nu}+\varphi, \mu \nu\right),
$$

where $D_{\mu}=\nabla_{\mu}+\omega_{\mu}, \omega_{\mu}=\psi_{, \mu}-\varphi_{, \mu}$. From equation (49) we can immediately read off the conformal anomaly for this case:

$$
T_{\mu}^{\mu}=\frac{1}{24 \pi}\left(R-6(\nabla \varphi)^{2}+4 \square \varphi+2 \square \psi\right) .
$$

The expression (69) for the conformal anomaly in a dilaton theory with general nonminimal coupling $\exp (-2 \varphi(\phi))$ to the scalars and for a general dilaton dependent norm involving $\exp (-2 \psi(\phi))$ was given first by the authors together with H. Liebl 12. For the special case $\operatorname{SRG}(\varphi=\psi=\phi)$ it appeared already in [7. However, another recent computation in the latter case [11] missed two important ingredients and therefore arrived at an incorrect result: the modification of the norm (65) and the omission of a null-mode for the compact manifold [46] upon which the computation of the anomaly had been performed. In the language of our much simpler approach, using well-known heat-kernel techniques 
(41], [38], [42]) with local scaling function the corresponding total derivatives cannot be missed. Although the situation was clarified soon 21] the incorrect factor in front of $\square \varphi=\square \psi=\square \phi$ from the last two terms in (69) 111] has been quoted and /or used in several papers [32. It is by no means "ambiguous" [1], [47.

The first term on the right hand side had been encountered already in the case of minimal coupling. Therefore, in the notation (61) in the conformal gauge

$$
T_{-+}^{(d i l)}=\frac{1}{12 \pi}\left[2 \partial_{+} \partial_{-} \varphi-3\left(\partial_{+} \varphi\right)\left(\partial_{-} \varphi\right)+\partial_{+} \partial_{-} \psi\right],
$$

and the contribution (63) to $T_{--}^{(n m)}$ becomes

$$
T_{--}^{(d i l)}=-\frac{1}{48 \pi} \int_{-\infty}^{z} d z^{\prime}\left(\partial_{z^{\prime}}-2 \partial_{z^{\prime}} \rho\right)\left[2 \partial_{z}^{2} \varphi-3\left(\partial_{z} \varphi\right)^{2}+\partial_{z}^{2} \psi\right]_{z^{\prime}} .
$$

\section{$4.3 \quad \Phi$-anomaly}

The variation of the effective action with respect to $\varphi$ or $\psi$ does not exhibit the same multiplicative property as the conformal variation, because after substituting in (43) the variation of $A^{(\mathrm{nm})}$ of (67) does not recombine to powers of $A^{(n m)}$. Therefore, the heat kernel technique is not applicable to the evaluation of (67) as it stands. However, crucial simplifications occur after relating it to flat space by means of a functional integral in conformal gauge $g_{\mu \nu}=e^{2 \rho} \eta_{\mu \nu}$. There one has $W^{(n m)}=W^{(n m)}(\rho, \phi)$ and thus the identities

$$
\begin{aligned}
\frac{\delta W^{(n m)}(\rho, \phi)}{\delta \phi} & =\int_{0}^{\rho} d \sigma \frac{\delta^{2} W^{(n m)}(\sigma, \phi)}{\delta \sigma \delta \phi}+\frac{\delta W^{(n m)}(0, \phi)}{\delta \phi} \\
\frac{\delta W^{(n m)}(0, \phi)}{\delta \phi} & =\frac{\delta W^{(n m)}(0, \varphi, \psi)}{\delta \varphi} \frac{d \varphi}{d \phi}+\frac{\delta W^{(n m)}(0, \varphi, \psi)}{\delta \psi} \frac{d \psi}{d \phi}
\end{aligned}
$$

are obvious. They relate the variation to the one at $\rho=0$, i.e. to a flat background. On the other hand, the first term on the right hand side of (72) can be expressed in terms of the trace of the EM tensor (conformal anomaly) by (42):

$$
\frac{\delta W^{(n m)}(\rho, \phi)}{\delta \phi}=-\int_{0}^{\rho} d \sigma \frac{\delta \sqrt{-g} T_{\mu}^{(n m) \mu}(\sigma, \phi)}{\delta \phi}+\frac{\delta W^{(n m)}(0, \phi)}{\delta \phi}
$$

Before dealing with the general case, we illustrate the problem in the simpler case $\varphi=\psi=\phi$ which is the relevant one for SRG.

Consider the last term in $(72)$ in detail. We note some restrictions on its possible form. In flat space $(\rho=0)$ the differential operator $A^{(n m)}(\sqrt{67})$ simplifies considerably:

$$
A^{(n m)}=-\eta^{\mu \nu} \partial_{\mu} \partial_{\nu}-E(\phi), \quad E(\phi)=\eta^{\mu \nu}\left(-\partial_{\mu} \phi \partial_{\nu} \phi+\partial_{\mu} \partial_{\nu} \phi\right)
$$


We denote by $\phi_{0}$ the background value (11) of $\phi, E\left(\phi_{0}\right)=E_{0}$, which in flat $D$-dimensional Minkowski space $(L=1)$ becomes

$$
E_{0}=\frac{2 a-1}{(2(1-a))^{2}} \frac{1}{u^{2}} .
$$

Since Minkowski space must be quantum stable, the effective action $W^{(n m)}(0, \phi)$ should have an extremum at $(\sqrt{76})$ with respect to arbitrary variations of $\phi$ and traceless variations of the metric:

$$
\left.\frac{\delta W^{(n m)}(0, \phi)}{\delta \phi}\right|_{E=E_{0}}=0,\left.\quad \frac{\delta W^{(n m)}(0, \phi)}{\delta g^{ \pm \pm}}\right|_{E=E_{0}}=0 .
$$

By changing $D$ between $D=4\left(a=\frac{1}{2}\right)$ and $D=\infty(a=1)$ one can obtain infinitely many values of the coefficient in front of $1 / u^{2}$ in (76). Hence, (77) must essentially hold for arbitrary value of that coefficient. This is a very strong condition. Some implications will be discussed below.

To evaluate the second term in (72), which at $\rho=0$ represents a flat space contribution $g_{\mu \nu}=\eta_{\mu \nu}$, for a general dilaton theory we rewrite $W^{(n m)}(0, \varphi, \psi)$ as

$$
W^{(n m)}(0, \varphi, \psi)=\frac{1}{4} \ln \int(d \vec{f}) \exp \left(-\int d^{2} x \sqrt{-\eta} \vec{f} \mathbf{1}_{2}(A) \vec{f}\right),
$$

where we have assumed that $\varphi$ and $\psi$ are now independent background fields. To compensate for double counting the degrees of freedom from introducing the two-component real field $\vec{f}$, an additional factor $1 / 2$ has been introduced. In flat space the integral in the exponential of (78) can be expressed as

$$
\int d^{2} x \sqrt{-\eta} \vec{f} \mathbf{1}_{2}(A) \vec{f}=\int d^{2} x \sqrt{-\eta} \vec{f} D D^{\dagger} \vec{f},
$$

where new differential operators $D=i \gamma^{\mu} e^{\psi} \partial_{\mu} e^{-\varphi}$ and $D^{\dagger}=D(\psi \leftrightarrow-\varphi)$ in spinor space have been introduced. Indeed, the right hand side of (79) is equal to

$$
\begin{array}{r}
\int d^{2} x \sqrt{-\eta}\left[\vec{f}\left(A+2 \gamma^{5} \epsilon^{\mu \nu} e^{2(\psi-\varphi)} \varphi,{ }_{\mu} \psi,_{\nu}\right) \vec{f}+\right. \\
\left.+\epsilon^{\mu \nu} e^{2(\psi-\varphi)} \varphi,{ }_{\mu} \partial_{\nu}\left(\vec{f} \gamma^{5} \vec{f}\right)\right],
\end{array}
$$

which may be used to prove (79) after integration by parts. Therefore, (78) becomes

$$
W^{(n m)}(0, \varphi, \psi)=\frac{1}{4} \ln \operatorname{det}\left(D D^{\dagger}\right) .
$$

For the $\zeta$-function of the operator $D D^{\dagger}$ we use its representation in terms of an inverse Mellin transform of the heat kernel

$$
\zeta_{D D^{\dagger}}(s)=\frac{1}{\Gamma(s)} \int_{0}^{\infty} d t t^{s-1} \operatorname{Tr} \exp \left(-t D D^{\dagger}\right) .
$$


This yields the variation of $\zeta$ with respect to $\varphi$ and $\psi$ :

$$
\begin{aligned}
\delta \zeta_{D D^{\dagger}}(s) & =\frac{1}{\Gamma(s)} \int_{0}^{\infty} d t t^{s-1} \operatorname{Tr} \sum \frac{(-t)^{n}}{n !}\left(2 \delta \psi\left(D D^{\dagger}\right)^{n}-2 \delta \varphi\left(D^{\dagger} D\right)^{n}\right) \\
& =\frac{2}{\Gamma(s)} \int_{0}^{\infty} d t t^{s} \operatorname{Tr}\left(-2 \delta \psi D D^{\dagger} \exp \left(-t D D^{\dagger}\right)+2 \delta \varphi D^{\dagger} D \exp \left(-t D^{\dagger} D\right)\right. \\
& =\frac{2 \Gamma(1+s)}{\Gamma(s)} \operatorname{Tr}\left(-2 \delta \psi D D^{\dagger}\left(-t D D^{\dagger}\right)^{-s-1}+2 \delta \varphi D^{\dagger} D\left(-t D^{\dagger} D\right)^{-s-1}\right) \\
& =-2 s \operatorname{Tr}\left(\left(D D^{\dagger}\right)^{-s} \delta \psi-\left(D^{\dagger} D\right)^{-s} \delta \varphi\right)
\end{aligned}
$$

Thus the introduction of $D D^{\dagger}$ has provided a means to achieve multiplicative factors for the two variations - at least in flat space, but this is sufficient for our purpose. By differentiating (83) with respect to $s$ one arrives at

$$
\begin{aligned}
\delta \zeta_{D D^{\dagger}}^{\prime}(0) & =-2\left(\zeta\left(0 \mid \delta \psi, D D^{\dagger}\right)-\zeta\left(0 \mid \delta \varphi, D^{\dagger} D\right)\right) \\
& =-2\left(a_{1}\left(\delta \psi, D D^{\dagger}\right)-a_{1}\left(\delta \varphi, D^{\dagger} D\right)\right) .
\end{aligned}
$$

To evaluate $a_{1}$ in the first term on the right hand side of (84) again the method of [39] is applicable. Introducing yet another type of differential operator in spinor space, we represent the operator $D D^{\dagger}$ as

$$
\begin{aligned}
D D^{\dagger} & =\left(\hat{g}^{\mu \nu} \mathcal{D}_{\mu} \mathcal{D}_{\nu}+\mathcal{E}\right), \\
\mathcal{D}_{\nu} & =\partial_{\nu}+\psi,{ }_{\nu}-\varphi,{ }_{\nu}-\gamma^{5} \epsilon^{\mu}{ }_{\nu} \varphi,{ }_{\mu}, \hat{g}^{\mu \nu}=e^{2(\psi-\varphi)} \eta^{\mu \nu}, \\
\mathcal{E} & =\hat{g}^{\mu \nu}\left(\hat{\nabla}_{\mu} \hat{\nabla}_{\nu} \varphi\right),
\end{aligned}
$$

and again use the general result (49). The covariant derivative $\hat{\nabla}_{\mu}$ refers to the present metric $\hat{g}_{\mu \nu}$. The second heat kernel coefficient $a_{1}$ for the operator $D^{\dagger} D$ is obtained by the simple replacement $\varphi \rightarrow-\psi, \psi \rightarrow-\varphi$.

Precisely at this point we should stress that the operator $D D^{\dagger}$ is only hermitian in the Dirac sense ( " $\gamma^{0}$-hermitian"). Nevertheless we see strong arguments in favour of our treatment from our result for the effective action (section 5).

From (84) with the flat d'Alembertian (Laplacian) $\Delta=\eta^{\mu \nu} \partial_{\mu} \partial_{\nu}$

$$
\delta \zeta_{D D^{\dagger}}^{\prime}(0)=-\frac{1}{3 \pi} \int d^{2} x \sqrt{-\eta}[\delta \psi(2 \Delta \varphi+\Delta \psi)+\delta \varphi(2 \Delta \psi+\Delta \varphi)]
$$

follows. We have retained the determinant also for the flat metric in order to cover the case of light coordinates (12) where $\eta=\operatorname{det} \eta \neq-1$. Also the covariantized version of eq. (86) can be obtained then very easily by means of the replacement $\sqrt{-\eta} \Delta \rightarrow \sqrt{-g} \square=\sqrt{-g} g^{\mu \nu} \nabla_{\mu} \nabla_{\nu}$.

Now all variations of the effective action $W^{(n m)}(\rho, \phi, \psi)$ with respect to all 
background fields can be summarized:

$$
\begin{aligned}
& \frac{\delta W^{(n m)}}{\delta \varphi}=-\frac{1}{12 \pi} \sqrt{-\eta}\left(6 \eta^{\mu \nu} \partial_{\nu}\left(\rho \partial_{\mu} \varphi\right)+2 \Delta \rho-2 \Delta \psi-\Delta \varphi\right), \\
& \frac{\delta W^{(n m)}}{\delta \psi}=-\frac{1}{12 \pi} \sqrt{-\eta}(\Delta \rho-2 \Delta \varphi-\Delta \psi), \\
& \frac{\delta W^{(n m)}}{\delta \rho}=-\frac{1}{12 \pi} \sqrt{-\eta}\left(-\Delta \rho-3 \eta^{\mu \nu}\left(\partial_{\mu} \varphi\right)\left(\partial_{\nu} \varphi\right)+2 \Delta \varphi+\Delta \psi\right) .
\end{aligned}
$$

For arbitrary functions $\varphi(\phi)$ and $\psi(\phi)$ in terms of the dilaton $\phi$, eqs. (72) and $(73)$ allow the evaluation of $T_{--}{ }^{(\phi)}$ in (64) for any dilaton theory. An important check of our calculation is provided by the integrability conditions $\delta^{2} W^{(n m)} / \delta \varphi \delta \rho=\delta^{2} W^{(n m)} / \delta \rho \delta \varphi$ etc. We take their validity as an a posteriori argument for the validity of our derivation of $\delta W / \delta \phi$. For $S R G$ the special choice $\varphi=\psi=\phi$ with (72), (73) yields

$$
\frac{1}{\sqrt{-g}} \frac{\delta W^{S R G}}{\delta \phi}=-\frac{1}{12 \pi}\left(6 \partial^{\mu}\left(\rho \partial_{\mu} \phi\right)+2 \square \rho-3 \square \phi\right) .
$$

\subsection{Radiative flux}

With (90) we are now in the position to calculate the contribution (64) to the flux. By simple partial integrations we find at finite $z$ or $u$ complete cancellation of the integral with the one in (63) (except for the total divergence there):

$$
T_{--}^{(\phi)}=-T_{--}^{(d i l)}+\frac{1}{16 \pi}\left[2\left(\partial_{z} \phi\right)\left(\partial_{z} \rho\right)+2 \rho\left(\partial_{z} \phi\right)^{2}+\left(\partial_{z} \phi\right)^{2}-\partial_{z}^{2} \phi\right]_{u_{h}}^{u}
$$

In the square bracket we have collected all the total derivatives. Eq. (91) yields the flux at any $u$. At $u \rightarrow \infty$ the asymptotic fluxes from $T_{--}^{(\phi)}$ and $T_{--}^{(d i t)}$ exactly cancel because the square bracket in (91) vanishes there due to the eq. (11). Thus, the flux at infinity exactly coincides with the one for minimal coupling [27]

$$
\left.T_{--}^{(n m)}\right|_{a s}=\left.T_{--}^{(m i n)}\right|_{a s}
$$

and the simple thermodynamical argument, presented in that connection 10] is also (at least superficially) applicable to the nonminimal case as well. It should be stressed that the cancellation and thus (92) is independent of the specific background, as along as the latter yields a vanishing contribution at $u \rightarrow \infty$ in the last term of (90). This result is highly welcome, because the integral for $T_{--}^{(d i l)}$ can be evaluated easily, say for $S R G$ from arbitrary $D>3$,

$$
T_{--}^{(d i l)}=-\frac{9}{2} \frac{D-2}{(D-1)} T_{--}^{(\min )},
$$

and its negative contribution by far outweighs $T_{--}^{(\min )}$. Of course, for a generalized dilaton theory with general nonminimal coupling $\varphi(\phi)$ in a general 
dilaton-dependent norm (65) with $\phi \rightarrow \psi(\phi)$ there may be choices of $\varphi$ and $\psi$ which allow $T_{--}^{(d i l)}=0$. For the simplest linear ansätze $\varphi=\alpha \phi, \psi=\beta \phi$ it has been shown in [12] that for certain relations between $\alpha$ and $\beta$ this is indeed the case. But as long as there is no valid argument for such a specific choice this had not been regarded an attractive alternative.

Now we turn to the flux at finite $u$, and especially to the region near the horizon $u \approx u_{h}$. Inserting (11) and (12) into the square bracket for finite values of $u$ (radius) yields a flux different from $T_{--}^{(\min )}$ :

$$
T_{--}^{(n m)}=T_{--}^{(m i n)}+\frac{1}{16 \pi} \frac{L^{2}}{u^{2}} \ln L
$$

We emphasize that - as in $T_{(--)}^{(\min )}$ - the condition of an Unruh vacuum "miraculously" produced a zero of second order at the horizon $\left(L \simeq\left(u-u_{h}\right)\right)$. However, in global coordinates (32) with factor $\left(u-u_{h}\right)^{-2}$ now a residual logarithmic divergence [4] is encountered. For this reason a more detailed discussion of the freedom given by renormalization of the one-loop conformal anomaly and of the $\phi$-anomaly is necessary, because this will affect the nonasymptotic region. The (regularized) divergent terms in the $\zeta$-function regularisation in (81) are given by $\zeta_{A}^{\prime}(0)$, and not by its variation as in (42).

In principle here two normalization parameters $\mu$ and $\mu^{\prime}$ are needed because two differential operators $\left(A\right.$ and $\left.D D^{\dagger}\right)$ appeared in our calculation:

$$
W^{(r e n)}=\ln \mu a_{1}(1, A)+\ln \mu^{\prime} a_{1}\left(1, D D^{\dagger}\right)
$$

From (49) it can be verified easily that the only term which is not a total derivative, in both terms of (95) for $\operatorname{SRG}(\varphi=\psi=\phi)$ becomes (with a convenient redefinition of $\mu$ )

$$
W_{(S R G)}^{(r e n)}=-\frac{\ln \mu}{16 \pi} \int d^{2} x \sqrt{-g}(\nabla \phi)^{2}
$$

The contribution to $T_{--}$is most easily found by interpreting $(96)$ as $\int d z\left(\partial_{z} \phi\right) \delta W / \delta \phi$ in the sense of the last term of (60), taking the conformal gauge where (96) is independent of $\rho$. Together with (94) the total flux at any (finite or infinite) $u$ becomes

$$
T_{--}^{(n m, r e n)}=T_{--}^{(n m)}+T_{--}^{(r e n)}=T_{--}^{(m i n)}+\frac{1}{16 \pi} \frac{L^{2}}{u^{2}} \ln \left(\frac{L}{\mu}\right) .
$$

Clearly no such renormalization term can arise from the flux for minimally coupled scalars. So (97) is the complete result for SRG. It does not depend on the explicit form of the Killing norm $L$, i.e.. it is true for a general background. Fixing $\mu$ at an arbitrary value $u_{0}$ of $u$ so that $L\left(u_{0}\right)=\mu$, at $u_{0}$ the renormalized flux coincides with the minimal one. In any case, the logarithmic divergence at the horizon in global coordinates cannot be eliminated. However, that singularity is an integrable one. It cannot lead to any "measured" infinity since 
an energy measurement should take a finite time. The main trouble with this singular term is that it does not seem to appear in full $D$-dimensional theory [10]. On the other hand, the form of the logarithm in (97) suggests a relation to renormalization. Indeed, such terms are typical for the effective action in a massless theory. In four-dimensional field theory a corresponding contribution would be $L^{4} \ln L$ which is harmless in the present context. The only exception is the case when the action is defined completely by an anomaly (e.g. the conformal one). In our calculations we had to use also the second anomaly (the " $\phi$-anomaly"). We may conjecture that the logarithmic term can be avoided if one uses the $D$-dimensional conformal coupling (cf. footnote 3 ) instead of the $D$-dimensional minimal one (3). Such a modification is needed anyhow in order to make the comparison with the Christensen and Fulling calculations [10] more direct.

\section{Effective Action}

The three eqs. (87)-(89) allow immediate functional integration of the complete effective action [27]

$$
\begin{aligned}
W^{(n m)}= & -\frac{1}{24 \pi} \int d^{2} x \sqrt{-\eta}\left(-\rho \Delta \rho+2 \psi \Delta \rho-\psi \Delta \psi-6 \rho\left(\partial_{\mu} \varphi\right)^{2}+\right. \\
& +4 \varphi \Delta \rho-4 \varphi \Delta \psi-\varphi \Delta \varphi),
\end{aligned}
$$

which can be written covariantly $(\sqrt{-\eta} \Delta \rightarrow \sqrt{-g} \square, \sqrt{-\eta} \Delta \rho=-\sqrt{-g} R / 2)$ as

$$
\begin{aligned}
W^{(n m)}= & -\frac{1}{24 \pi} \int d^{2} x \sqrt{-g}\left[-\frac{1}{4} R \square^{-1} R+3(\nabla \varphi)^{2} \square^{-1} R-2 R(\psi+\varphi)+\right. \\
& \left.+(\nabla \psi)^{2}+(\nabla \varphi)^{2}+4\left(\nabla^{\mu} \psi\right) \nabla_{\mu} \varphi\right]+W^{(r e n)}\left(\mu^{\prime}, \mu\right)
\end{aligned}
$$

The first term in (99) represents the Polyakov action [24] for minimal coupling $\varphi=\psi=0$ of the scalar fields. $\varphi(\phi)$ and $\psi(\phi)$ encode a general dilaton coupling of the scalars and of the dilaton-dependent measure, respectively. Thus eq. (99) generalizes the Polyakov action to the one for general non-minimal coupling to the dilaton field [27. The appearance of a nonlocal term should be emphasized. A functional integral applied to a bounded region in space time always contains ambiguities with respect to eventual surface variables. In that case (99) may acquire further (here undetermined) contributions. The term $W^{(r e n)}\left(\mu^{\prime}, \mu\right)$ depending on the renormalization points $\mu^{\prime}, \mu$ (as in (95)) has been discussed above for $S R G$. Also in the general case (99) it only receives contributions from $(\nabla \varphi)^{2}$ and $(\nabla \psi)^{2}$.

Again $S R G$ from $D$ dimensions $(\varphi=\psi=\phi)$ is of special interest:

$$
\begin{gathered}
W^{S R G}=\frac{1}{96 \pi} \int d^{2} x \sqrt{-g}\left[R \square^{-1} R-12(\nabla \phi)^{2} \square^{-1} R+12 \phi R-24(\nabla \phi)^{2}\right] \\
+W_{S R G}^{(r e n)}(\mu)
\end{gathered}
$$


The second, nonlocal term was not present in the analogous formula for the full effective action in [7]. The first three terms, however, appear in the "uncorrected" effective action there. In the first ref. [11] all four terms, but the last two with different factors, can be found. Similar actions were also used in refs. 32, 47.

Usually, the full effective action including the conformally invariant part is available as a power series in a small parameter 49. No such parameter exists for the BH background. Therefore, the closed form of our action (100) is essential. Two previous famous examples where such a closed form could be obtained were the Polyakov and WZNW actions. In those cases the effective actions were completely defined by the corresponding anomalies. It is remarkable that for general dilaton theories in $D=2$ we encounter a similar situation, because the " $\phi$-anomaly" (90) can be interpreted as carrying the information of part of the "complete" D-dimensional conformal anomaly.

Starting with [7] there have been further attempts to compute the flux component $T_{--}$from an effective action like (99), because it is natural to assume that a simple functional derivative with respect to the metric, as in the definition (20), applied to the effective action directly yields the desired result. However, we should recall that such a procedure is known to be a quite delicate matter [50]. Even without dilatons (as in the theory with minimal coupling) the proper choice of the asymptotics for the inverse d'Alembertian is very important in order to obtain correctly the (known) final outcome, already even for minimal coupling. In the presence of dilaton fields as in (100) we are not aware of any analysis which shows that there exists an appropriate choice at all.

This uncertainty reflects a basic weakness from which, in our opinion, all approaches are bound to suffer which use the existence of an integrated effective action as an essential intermediate step. Such an action describes a UV effect from quantum corrections, i.e. in coordinate space it is certainly only correct locally. This is also consistent with the rules for functional differentiation, which in an expression like (100) require sufficiently strong vanishing of the fields at the infinite boundaries of the integration in order to be able to perform partial integrations without surface contributions. But the region where the flux is needed here is precisely that boundary (at infinity)! There the flux, the functional derivative with respect to the metric, should give a nonvanishing result. Also the metric itself does not vanish there, but becomes Minkowskian. The main advantage of the approach used in our work 27] is that the input from the one loop quantum effects entered locally. The subsequent integral from the horizon to any value of $u$, including infinity is a trivial, well-defined ordinary one.

Several remarks on the validity of our method of evaluation of the effective action are important. The part depending on the conformal anomaly gives a well-defined local contribution to the Hawking flux (at least in our approach). To calculate $W^{(n m)}(0, \phi)$ we have added a total derivative to the classical action, thus replacing the operator $A$ by $D D^{\dagger}$. The latter operator is not hermitian with respect to the standard inner product for the scalar field and, therefore, corresponds to a different path integral measure. The effective actions calculated 
with $A$ and $D D^{\dagger}$ differ by an "anomaly" 5 . We believe that it is the operator $D D^{\dagger}$ that should be used in calculations of $W^{(n m)}(0, \phi)$ rather than $A$ itself. Our effective action evidently satisfies the conditions (77) because the only term which contributes to the variations, $(\nabla \phi)^{2}$, can be removed completely by adjusting the renormalization scale $\mu$. On the other hand, it is hard to see how (77) can be satisfied by the effective action for the operator $A=-\Delta-c / u^{2}$.

\section{Backscattering}

Our result (97) undoubtedly is suggestive, but it certainly requires further improvement. It is a central feature of the CF approach that the input is "minimal" in the sense that it explores the consequences of EM-conservation only. In the $D=2$ version even everything seems to be completely determined by the anomaly and there is, at first glance, no room for the inclusion of backscattering, an effect which in $D=4$ leads to complications [10], 50]. They are related to the replacement of the classical free-field equation $\partial_{+} \partial_{-} f=0$ for minimally coupled scalars in conformal gauge by the more complicated one

$$
\left(\partial_{+} u \partial_{-}+\partial_{-} u \partial_{+}\right) f=0 .
$$

The redefinition $f=\tilde{f} / \sqrt{u}$ as a consequence of (65), i.e. to $s$-wave amplitudes, yields

$$
\begin{aligned}
& \partial_{+} \partial_{-} \tilde{f}+V \tilde{f}=0, \\
& V=\frac{1}{2} \frac{\left(\partial_{+} u\right)\left(\partial_{-} u\right)}{u^{2}}-\frac{\partial_{+} \partial_{-} u}{u},
\end{aligned}
$$

describing the propagation of $s$-wave matter in a space-dependent "potential" (cf. (11), (12) for a fixed BH background):

$$
V=\frac{1}{4 u} L^{\prime} L-\frac{1}{8 u^{2}} L^{2}
$$

In their seminal paper 10 Christensen and Fulling computed the EM conservation in $D=4$ for spherical symmetry. There more undetermined functions remained in the $4 D$ EM-tensor when it had been restricted to spherical symmetry. The authors proposed to use the $4 D$ conformal anomaly and an estimate of backscattering effects in order to obtain information about those undetermined parts. In contrast, in the present $S R G$ approach everything seems to be fixed by the anomaly alone as long as (cf. Section 4.1) the assumption is made that the classical background of the scalar field vanishes $\left(f_{\text {class }}=0\right)$.

For minimally coupled scalars this assumption was consistent with the decoupling of $f_{\text {class }}$ from the problem of outgoing radiation: There a $\mathrm{BH}$ forms "immediately" from the incoming flux [51], and the (classical) BH remains stable $\left(T_{--}^{(\text {class })}=0\right.$ trivially follows from the condition $\left.\left.T_{--}^{(\text {class })}\right|_{u_{h}}=0\right)$.

\footnotetext{
${ }^{5}$ We are grateful to Dmitri Fursaev and Andrei Zelnikov for informing us about their calculations demonstrating that this "anomaly" is indeed non-zero.
} 
In the present case the period of the formation of the BH cannot be separated as cleanly from the situation after that. There will be still an outgoing flux of matter (as confirmed also by numerical computation [51]). Thus a solution for $f_{\text {class }} \neq 0$ to the coupled (classical) system of e.o.m.-s following from (2) together with (4) would be needed. The matter equation (in conformal gauge) would be (102). On that $f_{\text {class }}$ the quantum corrections are "riding". A more transparent (approximate) view of the situation is as follows: The flux of matter will influence the space curvature through the classical Einstein equation especially near the horizon by being backscattered from a maximum of some effective potential related to $V$ in 103 , which for $S R G$ at $D=4$ (with traceless EM-tensor, cf. footnote 3 ) is situated at $u \approx \frac{3}{2} u_{h}=3 M_{A D M}$ [10]. At infinity its contribution to $\left.T_{--}^{(n m)}\right|_{\text {as }}$ is negligible, but in the region $u_{h} \leq u \simeq 2 u_{h}$ it changes the geometric background to be used in the integration of the "EM-nonconservation", eq. (58). As a consequence, the flux at infinity will be attenuated by a certain amount ("grey factor"). We refer the reader to the extensive literature on this subject (e.g. [50, 51]).

The conclusion to be drawn for our present strictly $2 D(S R G)$ approach is that all these additional considerations can be (and should be) included as well in a purely $2 D$ approach. We do not see any obstacle for adapting the $4 D$ arguments to the $S R G$ setting directly. Of course, features specific for $4 D$ and related to the additional angular dependence will always remain outside the scope of a $2 D$ calculation.

\section{Comparison with other approaches, outlook}

The necessity to modify the energy-momentum tensor for non-minimally coupled scalars in order to avoid negative flux was recognized already in [7]. To this end attempts have been made [7, 26] to calculate the scale invariant non-local part of the effective action. Due to the ambiguities in definition of the inverse Laplacian this task is technically quite complicated. Usually such calculations are valid in a narrow region of the parameter space only, reflecting the weakness of the effective action approach. The effective action of [7] becomes singular in the limit of Minkowski space while the energy-momentum tensor of [26] fails to satisfy the conservation condition (58).

The importance of the modified conservation law (58) was noted in 25] where some modifications of the energy-momentum tensor were suggested.

The authors of the second ref. 44] proposed the introduction of two auxiliary fields, $\chi$ and $\psi$,

$$
\square \chi=(\nabla \phi)^{2}, \quad \square \psi=R,
$$

to avoid non-locality in the effective action. Arbitrary constants arising from solving the equations (105) for $\chi$ and $\psi$ were used later by Balbinot and Fabbri [48 to model different vacuum states. In particular, they obtained that the conformal anomaly induced effective action either produces negative Hawking 
flux or gives logarithmic behaviour at the horizon as in our eq. (94). We can even further sharpen this result. Indeed, since the fields $\chi$ and $\psi$ were used to represent the non-local term $\int(\nabla \phi)^{2}(1 / \square) R$ in two different ways, a consistency condition must hold

$$
\int d^{2} x \sqrt{-g}(\nabla \phi)^{2} \psi=\int d^{2} x \sqrt{-g} R \chi .
$$

This condition turns out to be very restrictive. For example, it immediately gives $C=0$ in the notations of [48]. From eq. (13) of [48] it is seen that it is not possible to remove the logarithmic term from the energy-momentum tensor.

Our approach has successfully passed various consistency tests and gives physically plausible results. Namely, the Hawking flux is positive and the Hawking temperature is defined by the surface gravity in the usual way. However, we should mention certain weak points of this approach as well. The first problem is common for nearly all approaches dealing with non-minimal coupling in 2D. This is the presence of the $L^{2} \ln L$ term in the energy momentum tensor near the horizon. In our view this singularity is quite mild and may be an artefact of the $2 \mathrm{D}$ renormalization scheme. Possibly it can be removed by using the $D$ dimensional conformal action for scalars. On the other hand, we are not aware of any $D=4$ calculation which explicitly forbids such a term. A more serious problem for us is to justify the transition to the operator $D D^{\dagger}$ in the calculation of $W^{(n m)}(0, \phi)$. Even though we have presented certain arguments in favour of this choice, as compared to direct calculations with the operator $A$, the whole procedure still involves a step which lacks complete mathematical rigour.

In the present paper we have critically reviewed various approaches to Hawking radiation from $2 \mathrm{D}$ black holes and presented an approach which, in our opinion, so far is the most appropriate one for a proper description of this process, although we must admit that this problem cannot be considered to be settled as yet. To clarify the situation a direct calculation starting from $4 \mathrm{D}$ quantum theory with $4 \mathrm{D}$ renormalization conditions is needed urgently.

Many questions of black hole physics are outside the scope of the present review. For more information an interested reader should consult the large literature on the subject from which we recommend for a recent collection ref. [52].

\section{Acknowledgements}

The authors are grateful for valuable discussions and/or correspondence with R. Balbinot, M. Bordag, G. Cognola, A. Fabbri, D. Fursaev, J. Louko, V. Mukhanov, A. Wipf and A. Zelnikov. We thank F. Hehl for his kind invitation to write this review. This work has been supported by Fonds zur Förderung der wissenschaftlichen Forschung (Austrian Science Foundation), Project P 12.815TPH, the Alexander von Humboldt Foundation and RFBR, grant 97-01-01186.

\section{References}


[1] P. Thomi, B. Isaak and P. Hajicek, Phys. Rev. D 30 (1984) 1168; P. Hajicek, Phys.Rev. D 30 (1984) 1168.

[2] G. Mandal, A. Sengupta, and S.R. Wadia, Mod. Phys. Lett A 6 (1991) 1685; V.P. Frolov, Phys. Rev. D 46 (1992) 5383; J.G. Russo and A.A. Tseytlin, Nucl. Phys. B 382 (1992) 259; J.G. Russo, L. Susskind and L. Thorlacius, Phys. Lett. B 292 (1992) 13; T. Banks, A. Dabholkar, M. Douglas and M. O'Loughlin, Phys. Rev D 45 (1992) 3607; S.P. deAlwis, Phys. Lett. B 289 (1992) 278; S.D. Odintsov and I.L. Shapiro, Phys. Lett. B 263 (1991) 183; E. Elizalde, P. Fosalba-Vela, S. Naftulin and S.D. Odintsov, Phys. Lett. B 352 (1995) 235.

[3] S. Elitzur, A. Forge and E. Rabinovici, Nucl. Phys. B 359 (1991) 581; E. Witten, Phys. Rev. D 44 (1991) 314; C.G. Callan, S.B. Giddings, J.A. Harvey and A. Strominger, Phys. Rev. D 45 (1992) 1005.

[4] M.O. Katanaev, W. Kummer and H. Liebl, Phys. Rev. D 53 (1996) 5609; M.O. Katanaev, W. Kummer and H. Liebl, Nucl. Phys. B 486 (1997) 353.

[5] W. Kummer, H. Liebl and D.V. Vassilevich, Nucl. Phys. B 493 (1997) 491; ibid. B 513 (1998); 723; ibid. B 544 (1999) 403.

[6] W. Kummer and D.V. Vassilevich (in preparation)

[7] V. Mukhanov, A. Wipf and A. Zelnikov, Phys. Lett. B 332 (1994) 283.

[8] S.W. Hawking, Commun. Math. Phys. 43 (1975) 199.

[9] W.G. Unruh, Phys. Rev. D 14 (1976) 870.

[10] S.M. Christensen and S.A. Fulling, Phys. Rev. D 15 (1977) 2083; P. Candelas, Phys. Rev. D 21 (1980) 2185.

[11] R. Bousso and S.W. Hawking, Phys. Rev. D 56 (1997) 7788.

[12] W. Kummer, H. Liebl and D.V. Vassilevich, Mod. Phys. Lett. A 12 (1997) 2683.

[13] T. Chiba and M. Siino, Mod. Phys. Lett. A 12 (1997) 2083; S. Ichinose, Phys. Rev. D 57 (1998) 6224.

[14] C.Teitelboim, Phys. Lett. 126 B (1983) 41; R. Jackiw, Quantum Theory of Gravity, ed. S. Christensen p. 403. Hilger, Bristol 1984.

[15] S. Mignemi, Phys. Rev. D 50 (1994) R4733.

[16] A. Fabbri and J.G. Russo, Phys. Rev. D 53 (1996) 6995.

[17] J.S. Lemos and P.M. Sa, Phys. Rev. D 49 (1994) 2897.

[18] R. Arnowitt, S. Deser and C. W. Misner, in Gravitation: An Introduction to Current Research, ed. L. Witten, Wiley, New York 1962. 
[19] W. Kummer and D.J. Schwarz, Phys. Rev. 45 (1992) 3628; P. Schaller and T. Strobl, Class. Quant. Grav. 11 (1994) 331; T. Strobl, Phys. Rev. D 50 (1994) 7346; W. Kummer and P. Widerin, Phys. Rev. D 52 (1995) 6965; T. Klösch and T. Strobl, Class. Quant. Grav. 13 (1996) 965 and 14 (1997) 1689; W. Kummer and G. Tieber, Phys. Rev. D 59 (1999) 044001.

[20] H. Liebl, D.V. Vassilevich and S. Alexandrov, Class. Quant. Grav. 14 (1997) 889.

[21] W. Kummer, H. Liebl and D.V. Vassilevich, Phys. Rev. D 58 (1998) 108501.

[22] S.A. Fulling, Phys. Rev. D 7 (1973) 2850; P.C.W. Davies, J. Phys. A 8 (1975) 609.

[23] J.B. Hartle and S.W. Hawking, Phys. Rev. D 13 (1976) 2188.

[24] A.M. Polyakov, Phys. Lett. B 103 (1981) 207.

[25] R. Balbinot and A. Fabbri, Phys. Rev. D 59 (1999) 044031.

[26] F.C. Lombardo, F.D. Mazzitelli and J.G. Russo, Phys. Rev. D 59 (1999) 064007..

[27] W. Kummer and D.V. Vassilevich, Effective action and Hawking radiation for dilaton coupled scalars in two dimensions (hep-th/9811092), to be published in Phys. Rev. D

[28] D. Louis-Martinez, Phys. Rev. D 55 (1997) 782; M. Navarro, Phys. Rev. D 56 (1997) 2384; Y. Kiem, C.-Y. Lee and D. Park, Class. Quant. Grav. 15 (1998) 2973; J. Cruz, J.M. Izquierdo, D.J. Navarro and J. Navarro-Salas, Phys. Rev. D 58 (1998) 044010; J. Cruz and J. Navarro-Salas, Mod. Phys. Lett. A 12 (1997) 2345; G. Kunstatter, R. Petryk and S. Shelemy, Phys. Rev. D 57 (1998) 3537; M. Cavaglia, Phys. Rev. D 59 (1999) 084011; J. Cruz, A. Fabbri and J. Navarro-Salas, Phys. Lett. B 449 (1999) 30; S. Cassemiro F.F., V.O. Rivelles, Canonical and functional Schrödinger quantization of two-dimensional dilaton gravity (hep-th/9812096).

[29] K.V. Kuchař, J. D. Romano and M. Varadarajan, Phys. Rev. D.. 55 (1997) 795; D. Cangemi and R. Jackiw, Phys. Lett. B 337 (1994) 271; D. Cangemi and R. Jackiw and D. Zwiebach, Ann. Phys. 245 (1996) 408.

[30] E. d'Hoker, D.Z. Freedman and R. Jackiw, Phys. Rev. D. 28 (1983) 2583; R. Jackiw, Nucl. Phys. B 252, (1985) 343; D. Cangemi and R. Jackiw, Phys. Rev. Lett 69 (1991) 233; R.B. Mann, A. Shiekh and L. Tarasov, Nucl. Phys. B 341 (1990) 134; D. Banks and M. O'Loughlin, ibid. B 362 (1991) 649; H.J. Schmidt, J. Math. Phys. 32 (1991) 1562; S.D. Odintsov and I.J. Shapiro, Phys. Lett. B 263 (1991) 183, Mod. Phys. Lett. A 7 (1992) 437; V.P. Frolov, Phys. Rev. D 46 (1992) 5383; J.G. Russo and A.A. Tseytlin, Nucl. Phys. B 382 (1992) 259; I.V. Volovich, Mod. Phys. 
Lett. A 8 (1992) 1827; R.P. Mann, Phys. Rev. D 47 (1993) 4438; D. LouisMartinez, J. Gegenberg and G. Kunstatter, Phys. Lett. B 321 (1994), 193; D. Louis-Martinez and G. Kunstatter, Phys. Rev. D 49 (1994) 5227.

[31] cf. e.g. R.G. Wald, General Relativity, Univ. of Chicago Press 1984.

[32] R. Bousso and S. W. Hawking, Phys. Rev. D 57 (1998) 2436; S. Nojiri and S.D. Odintsov, Mod. Phys. Lett. A 12 (1997) 2083 and Phys. Rev. D 57 (1998) 2363; E. Elizalde, S. Nojiri and S.D. Odintsov, Possible quantum instability of primordial black holes (hep-th/9901026).

[33] K.C.K. Chan, J.D.E. Creighton and R.B. Mann, Phys. Rev. D 54 (1996) 3892; K.C.K. Chan, Is mass conformally invariant?, gr-qc/9701029.

[34] V.A. Fock, The theory of space, time, and gravitation, Pergamon Press, Oxford, 1964; L.D. Faddeev, Uspekhi Fiz. Nauk [Sov. Phys. Uspekhi] 136 (1982) 435.

[35] J. Cruz, A. Fabbri and J. Navarro-Salas, Phys. Lett. B 449 (1999) 30.

[36] D.G. Boulware, Phys. Rev. D 13 (1976) 2169.

[37] W. Israel, Phys. Lett. 57 A (1976) 107; G.W. Gibbons and M.J. Perry, Phys. Rev. Lett. 36 (1976) 985.

[38] M.F. Atiyah, V.K. Patodi and I.M. Singer, Math. Proc. Camb. Phil. Soc. 79 (1976) 71.

[39] P.B. Gilkey, J. Diff. Geom. 10 (1975) 601; Invariance Theory, the Heat Equation, and the Atiyah-Singer Index Theorem, CRC Press, Boca Raton 1994.

[40] cf.e.g. M.E. Peskin and D.V. Schroeder, An Introduction to Quantum Field Theory, Addison Wesley 1995.

[41] J.S. Dowker and R. Critchley, Phys. Rev. D 13 (1976) 3224; S.W. Hawking, Commun. Math. Phys. 55 (1977)133.

[42] G. Esposito, A.Y. Kamenshchik and G. Pollifrone, Euclidean Quantum Gravity on Manifolds with Boundary, Kluwer, Dordrecht 1997.

[43] W.T. Kim, Phys. Rev. D 60 (1999) 024010; W.T. Kim and J.J. Oh, "Dilaton driven Hawking radiation in $\operatorname{AdS}(2)$ black hole," hep-th/9905007.

[44] D. Grumiller and W. Kummer, Absolute conservation law for black holes, TUW-99-02 (gr-qc/9902074).

[45] M.O. Katanaev, T. Klösch and W. Kummer, Global properties of warped solutions in general relativity gr-qc/9807079), to be published in Ann. of Phys. 
[46] J.S. Dowker, Class.Quant.Grav. 15 (1998) 1881.

[47] A. Miković and V. Radovanović, Class. Quant. Grav. 15 (1998) 827; M. Burić, A. Miković and V. Radovanović, Phys. Rev. D 59 (1998) 084002.

[48] R. Balbinot and A. Fabbri, 4D quantum black hole physics from 2D models? (hep-th/9904034).

[49] G.A. Vilkovisky, in Quantum Theory of Gravity (ed. S.M. Christensen) Hilger, Bristol 1984; A.O. Barvinsky and G.A. Vilkovisky, Nucl. Phys. B 282 (1987) 163, ibid. B 333 (1990) 471; Yu. Gusev and A. Zelnikov, Class. Quant. Grav. 15 (1998) 13.

[50] I.D. Novikov and V.P. Frolov, Physics of Black Holes Kluwer, Dordrecht 1989.

[51] M.W. Choptuik, Ph.D. Thesis, Univ. of Brit. Columbia (1986), Phys. Rev. D 44 (1991) 3124

[52] F.W. Hehl, R.J.K. Metzler and C. Kiefer, (eds.), Black Holes: Theory and Observation, Proc. W.E. Heraeus Seminar, (Springer, Berlin 1998). 